\section{$\underset{\substack{\text { hommes } \\ \text { \& migrations }}}{ }$}

\section{Hommes \& migrations}

Revue française de référence sur les dynamiques

migratoires

\section{$1308 \mid 2014$}

Les Paris des migrants

\title{
Populations d'origine sud-asiatique à Paris et en Ile-de-France \\ Distribution et visibilité
}

\section{Delon Madavan}

\section{(2) OpenEdition \\ 1 Journals}

\section{Édition électronique}

URL : http://journals.openedition.org/hommesmigrations/2991

DOI : 10.4000/hommesmigrations.2991

ISSN : 2262-3353

\section{Éditeur}

Musée national de l'histoire de l'immigration

\section{Édition imprimée}

Date de publication : 1 octobre 2014

Pagination : $33-43$

ISBN : 978-2-919040-29-2

ISSN : $1142-852 X$

\section{Référence électronique}

Delon Madavan, «Populations d'origine sud-asiatique à Paris et en lle-de-France », Hommes \& migrations [En ligne], 1308 | 2014, mis en ligne le 01 octobre 2017, consulté le 19 avril 2019. URL: http://journals.openedition.org/hommesmigrations/2991; DOI : 10.4000/hommesmigrations.2991 


\title{
POPULATIONS D'ORIGINE SUD-ASIATIQUE À PARIS ET EN ÎLE-DE-FRANCE DISTRIBUTION ET VISIBILITÉ
}

par DELON MADAVAN, géographe et post-doctorant au Centre d'études et de recherche sur l'Inde, l'Asie du Sud et sa diaspora, de l'université du Québec à Montréal (CERIAS/UQÀM).

\author{
Depuis quarante ans, certains quartiers des $X^{\mathrm{e}}$ et \\ XVIIIe arrondissements de Paris ont la préférence des migrants \\ originaires d'Asie du Sud. S'ils continuent de fréquenter \\ le nord de la capitale, leurs stratégies résidentielles, \\ qui évoluent en fonction de plusieurs paramètres comme \\ I'ancienneté de leur migration ou leur statut familial, \\ les conduisent désormais en banlieue. Les quartiers \\ de La Chapelle et de la gare de l'Est demeurent néanmoins \\ des territoires offrant des lieux de sociabilité privilégiés.
}

Si la présence des populations d'origine sud-asiatique $^{1}$ en France (tableau 1, 2 et 3) n'est pas un phénomène récent ${ }^{2}$, elle se développe surtout à partir des années 1970. Il est possible de distinguer les parcours migratoires liés au passé colonial des migrants avec la France et ceux arrivés du Sri Lanka, d'Inde, du Pakistan et du Bangladesh (figure 1).

La France est un choix de migration privilégié pour les communautés sud-asiatiques issues de territoires qu'elle a colonisés, d'anciens comptoirs français en Inde et en Indochine, d'actuels DOM (Réunion, Guadeloupe et Martinique), de l'île Maurice et de Madagascar. La présence de ces citoyens français d'origine sud-asiatique, qui ont joué le rôle d'intermédiaires, a favorisé l'implantation de nouvelles vagues d'immigrants issus de régions du souscontinent indien colonisées par les Britanniques (Sri Lanka, Inde, Pakistan, Bangladesh).

Le durcissement des lois d'immigration en GrandeBretagne depuis 1962 (Immigration Act en 1971) pour réduire l'afflux de migrants du Commonwealth force les populations du sous-continent indien à se tourner vers d'autres destinations. Si, parmi ces derniers, certains sont arrivés en France pour pour- 
/// Tableau 1 : Immigrés selon le pays de naissance et la nationalité en France en $2006^{3}$

\begin{tabular}{|c|c|c|c|}
\hline PAYS DE NAISSANCE & FRANÇAIS PAR ACQUISITION & ÉTRANGERS & TOTAL \\
\hline Île Maurice & 18950 & 11000 & 29950 \\
\hline Bangladesh & 700 & 2600 & 3300 \\
\hline Inde & 15900 & 11800 & 27700 \\
\hline Pakistan & 5300 & 11000 & 300 \\
\hline Sri Lanka & 12100 & 20800 & 32900 \\
\hline
\end{tabular}

Source : Insee, Recensement général de la population (RGP) 2006 ; Christine Moliner, "Invisible et modèle ? Première approche de l’immigration sud-asiatique en France", rapport d'étude pour la Direction de l'accueil, de l'intégration et de la citoyenneté, ministère de l'Immigration, de l'Intégration, de l'Identité nationale et du Développement solidaire, Paris, 2009, p. 11.

/// Tableau 2 : Immigrés selon le pays de naissance en France métropolitaine en 2011

\begin{tabular}{|c|c|}
\hline PAYS DE NAISSANCE & TOTAL \\
\hline Île Maurice & 31508 \\
\hline Inde & 32730 \\
\hline Pakistan & 19736 \\
\hline Sri Lanka & 39726 \\
\hline
\end{tabular}

Source : Insee, Recensement général de la population (RGP), 2011.

/// Tableau 3 : Estimation du poids démographique des communautés d'origine sud-asiatique proposée par les chercheurs ${ }^{4}$

\begin{tabular}{|c|c|}
\hline COMMUNAUTÉS D'ORIGINE SUD-ASIATIQUE & ESTIMATION \\
\hline Sri lankais & 80000 à 100000 \\
\hline Indiens & 70000 à 100000 \\
\hline Pakistanais & 50000 \\
\hline Indo-Mauriciens & 50000 \\
\hline Bangladeshis & 10000 (estimation établie par plusieurs enquêtes) \\
\hline
\end{tabular}

Source : Christine Moliner, “Invisible et modèle ? Première approche de l'immigration sud-asiatique en France”, op. cit., p.11.

3. Le recensement de 2006 nous permet d'avoir une idée de la proportion des principaux groupes nationaux d'origine sud-asiatique en France. Les chiffres sur les immigrés du sous-continent indien, qui incluent les Sud-Asiatiques ayant gardé la nationalité de leur pays d'origine et ceux devenus français, permettent de constater que les principaux foyers d'émission sont dans l'ordre : le Sri Lanka, I'Inde et le Pakistan. Ces données ont aussi l'intérêt de souligner qu'un nombre non négligeable de ces immigrés, ayant répondu au recensement, ont obtenu depuis leur arrivée la nationalité française par acquisition : 57,4\% des immigrés venus d'Inde, 36,8 \% de ceux venus de Sri Lanka et 32,5\% de ceux venus du Pakistan. 
suivre leurs études ou comme travailleurs qualifiés, les premières vagues d'immigrants sont souvent venues clandestinement. De nombreux Tamouls sri lankais, et dans une moindre mesure des sikhs et des Bangladeshis issus des minorités religieuses, ont vu leur situation régularisée grâce à l'octroi de l'asile politique par l'Office français de protection des réfugiés et apatrides (Ofpra). Les mesures de régularisation adoptées par le gouvernement français ont également permis de sortir de la précarité de nombreux Pakistanais, Mauriciens et Indiens. L'immigration de personnes d'origine sud-asiatique se poursuit aujourd'hui sous différentes formes (légales ou non). La procédure de regroupement familial, les mariages transnationaux avec un partenaire du pays d'origine, les études et le recrutement par des entreprises basées en France de travailleurs qualifiés sud-asiatiques continuent d'alimenter ces flux migratoires vers la France.

\section{L'ancrage des immigrants d'origine sud-asiatique à Paris}

Plusieurs facteurs expliquent l'implantation des membres de ces communautés le long de la rue du Faubourg-Saint-Denis. La présence des deux grandes gares parisiennes (gare du Nord et gare de l'Est), qui étaient des portes d'entrée pour les premières vagues de migrants du sous-continent indien, est une première raison. Les immigrés d'origine sud-asiatique se sont installés à proximité d'autres quartiers populaires (Barbès, Goutte-d'Or et Château-Rouge) abritant des vagues d'immigration plus anciennes (Maghreb et Afrique subsaha- rienne) et des ouvriers. La forte concentration dans ces deux arrondissements d'hôtels modestes et peu coûteux a permis aux premiers immigrés d'Asie du Sud de s'établir plus aisément seuls sans relais communautaire.

L'ouverture, dans les années 1960, des premiers restaurants et commerces sud-asiatiques (épiceries, magasins de tissus) par des marchands marécars musulmans (originaires des anciens établissements français en Inde) a également joué un rôle important dans l'installation des vagues ultérieures d'immigration sud-asiatique.

Les premiers établissements Les immigrés d'origine marchands marécars, rue Clauzel, près du métro SaintGeorges, dans le XIX arrondissement de Paris, puis l'expansion spatiale de leurs commerces en direction de la rue du Paradis et de la rue du Faubourg-Saint-Denis ont facilité l'installation des sud-asiatique se sont installés à proximité d'autres quartiers populaires (Barbès, Goutte-d'Or et Château-Rouge) abritant des vagues d'immigration plus anciennes (Maghreb et Afrique subsaharienne) et des ouvriers.

Pakistanais, des Mauriciens et des Tamouls sri lankais ${ }^{5}$. Les Marécars, qui sont les premiers employeurs ethniques sud-asiatiques en France, favorisent notamment l'installation des Indo-Mauriciens et des Pakistanais dans le Sentier grâce à l'hébergement et à l'emploi qu'ils leur fournissent ${ }^{6}$. Le recrutement de nombreux Pakistanais dans les industries de l'habillement du Sentier et des quartiers avoisinants participe également à la concentration spatiale des membres de cette communauté dans le Xe arrondissement ${ }^{7}$. Par la suite, les entrepreneurs pakistanais, mauriciens et nord-indiens développent de nouvelles activités 
économiques (épiceries, restaurants) le long de la portion de la rue du Faubourg-Saint-Denis, située entre la gare de l'Est et la station de métro Strasbourg-Saint-Denis.

En 2011, 79,5\% des immigrés

indiens, pakistanais et sri lankais résidant en France métropolitaine habitent dans la région parisienne. En Île-de-France, seuls 13 \% des immigrés indiens, pakistanais et sri lankais vivent à Paris.

métro La Chapelle. Avec la déoradation de la situation politique au Sri Lanka ${ }^{9}$ dans les années 19801990, de plus en plus de Tamouls fuyant la guerre se réfugient en France. On assiste en parallèle à une multiplication des entreprises tamoules sri lankaises dans le $\mathrm{X}^{\mathrm{e}}$ arrondissement et à la création d'un quartier commercial tamoul au bout de plusieurs années.

\section{L'évolution des statégies résidentielles des communautés d'origine sud-asiatique}

Si les premières vagues d'immigrants d'origine sudasiatique se sont établies dans les Xe et XVIII arrondissements, les stratégies résidentielles ont évolué au fil des années. Les membres de cette communauté ont, pour beaucoup, préféré quitter la capitale pour sa banlieue.

Les données statistiques officielles sur la distribution des populations d'origine sud-asiatique à Paris et en Île-de-France ne donnent, pour plusieurs raisons, qu'une vision très approximative de leur poids démographique. Ainsi, les recensements de 1999 de l'Institut national de la statistique et des études économiques (Insee) ne permettent d'appréhender que la présence des étrangers indiens, pakistanais et sri lankais (en situation régulière) à
Paris et dans ses différents arrondissements. Les autres groupes étrangers d'origine sud-asiatique (Bangladesh, Maldives) ne bénéficient pas d'une entrée spécifique, leur effectif étant jugé insuffsant. Les données sur les Mauriciens n'ont, par contre, pas été retenues car les recensements français regroupent dans cette entrée toutes les communautés originaires de cette île de l'océan Indien (Indiens, Créoles, Chinois, Franco-Mauriciens). Enfin, le recensement de l'Insee de 1999 ne permet pas d'appréhender la distribution spatiale des Domiens d'origine sud-asiatique, des deuxième et troisième générations nées en France ou encore des clandestins. Malgré ces manques, l'analyse de la distribution des étrangers indiens, pakistanais et sri lankais à Paris et par arrondissement en 1999 (tableau 4) révèle que les membres de ces communautés se concentrent essentiellement dans les arrondissements populaires du nord-est de la capitale. Il est d'ailleurs intéressant de noter que $59 \%$ de ces personnes habitent dans les $\mathrm{X}^{\mathrm{e}}, \mathrm{XI}^{\mathrm{e}}$, XVIII', $\mathrm{XIX}^{\mathrm{e}}$ ou XXe arrondissements. Le XVIII est d'ailleurs de loin l'arrondissement qui compte le plus d'étangers indiens, pakistanais et sri lankais.

Les données statistiques du recensement de l'Insee de 2011 (tableau 5) sur les immigrés indiens, pakistanais et sri lankais à Paris, en Île-de-France et en France métropolitaine apportent des éléments d'informations supplémentaires sur la distribution géographique de ces groupes sud-asiatiques. En 2011, 79,5 \% des immigrés indiens, pakistanais et sri lankais résidant en France métropolitaine habitent dans la région parisienne. En Île-de-France, seuls $13 \%$ des immigrés indiens, pakistanais et sri lankais vivent à Paris. La majorité des immigrés de ces trois groupes vivant en région parisienne habitent en banlieue. La Seine-SaintDenis, département le plus cosmopolite de France métropolitaine, est de loin celui où vivent le plus d' immigrés sud-asiatiques, soit un tiers d'entre eux (et même $40 \%$ dans le cas des immigrés sri lankais) vivent dans ce département. 
La carte de la distribution des associations tamoules sri lankaises en région parisienne selon la localisation du siège social confirme aussi que les membres de cette communauté préfèrent s'installer dans les villes de la banlieue nord-est de Paris (figure 2). D’après une recherche menée par Gaëlle Dequirez dans le Journal officiel, " $85 \%$ des associations tamoules sri lankaises recensées déclarent leur siège en région parisienne. Le département le plus habité par les Tamouls est la Seine-Saint-Denis : on y retrouve le quart des associations tamoules sri lankaises de France. Puis viennent le Val-de-Marne et le Val-d'Oise ${ }^{10}$.

Tableau 4 : Les étrangers indiens, pakistanais et sri lankais à Paris et par arrondissement en 1999

\begin{tabular}{|c|c|c|c|c|c|c|c|c|c|c|c|}
\hline \multirow[t]{2}{*}{ NATIONALITÉS } & \multirow[t]{2}{*}{ PARIS } & \multicolumn{10}{|c|}{ ARRONDISSEMENTS } \\
\hline & & 1 & 2 & 3 & 4 & 5 & 6 & 7 & 8 & 9 & 10 \\
\hline Indiens & 1420 & 13 & 21 & 10 & 6 & 18 & 14 & 43 & 18 & 31 & 147 \\
\hline Pakistanais & 1102 & 1 & 71 & 14 & 0 & 1 & 2 & 4 & 9 & 24 & 142 \\
\hline Sri lankais & 6216 & 21 & 28 & 68 & 33 & 21 & 23 & 89 & 66 & 90 & 492 \\
\hline Total & 8738 & 35 & 120 & 92 & 39 & 40 & 39 & 136 & 93 & 145 & 781 \\
\hline
\end{tabular}

\begin{tabular}{|c|c|c|c|c|c|c|c|c|c|c|c|}
\hline \multirow[t]{2}{*}{ NATIONALITÉS } & \multirow[t]{2}{*}{ PARIS } & \multicolumn{10}{|c|}{ ARRONDISSEMENTS } \\
\hline & & 11 & 12 & 13 & 14 & 15 & 16 & 17 & 18 & 19 & 20 \\
\hline Indiens & 1420 & 13 & 21 & 10 & 6 & 18 & 14 & 43 & 18 & 31 & 147 \\
\hline Pakistanais & 1102 & 1 & 71 & 14 & 0 & 1 & 2 & 4 & 9 & 24 & 142 \\
\hline Sri lankais & 6216 & 21 & 28 & 68 & 33 & 21 & 23 & 89 & 66 & 90 & 492 \\
\hline Total & 8738 & 35 & 120 & 92 & 39 & 40 & 39 & 136 & 93 & 145 & 781 \\
\hline
\end{tabular}

Source : Insee, Recensement général de la population (RGP), 1999

/// Tableau 5 : Les immigrés indiens, pakistanais et sri lankais en île-de-France en 2011

\begin{tabular}{|c|c|c|c|c|c|c|}
\hline NATIONALITÉS & PARIS & $\begin{array}{c}\text { HAUTS-DE- } \\
\text { SEINE }\end{array}$ & $\begin{array}{c}\text { SEINE- } \\
\text { SAINT-DENIS }\end{array}$ & $\begin{array}{c}\text { VAL-DE- } \\
\text { MARNE }\end{array}$ & VAL-D'OISE & ESSONNE \\
\hline Sri lankais & 5154 & 2728 & 13962 & 4133 & 3473 & 1419 \\
\hline Indiens & 3095 & 1864 & 6252 & 1944 & 4383 & 2902 \\
\hline Pakistanais & 1383 & 608 & 5261 & 1207 & 4321 & 878 \\
\hline Total & 9632 & 5200 & 25475 & 7284 & 12177 & 5199 \\
\hline
\end{tabular}

\begin{tabular}{|c|c|c|c|c|}
\hline NATIONALITÉS & SEINE-ET-MARNE & YVELINES & ÎLE-DE-FRANCE & $\begin{array}{c}\text { FRANCE } \\
\text { MÉTROPOLITAINE }\end{array}$ \\
\hline Sri lankais & 2704 & 1129 & 34702 & 39726 \\
\hline Indiens & 1522 & 1269 & 23232 & 32730 \\
\hline Pakistanais & 1109 & 543 & 15312 & 19736 \\
\hline Total & 5335 & 2941 & 73246 & 92192 \\
\hline
\end{tabular}

Source : Insee, Recensement général de la population (RGP), 2011. 
/// Figure 1 : Foyers d'émigration des principales communautés d'origine sud-asiatique à Paris.
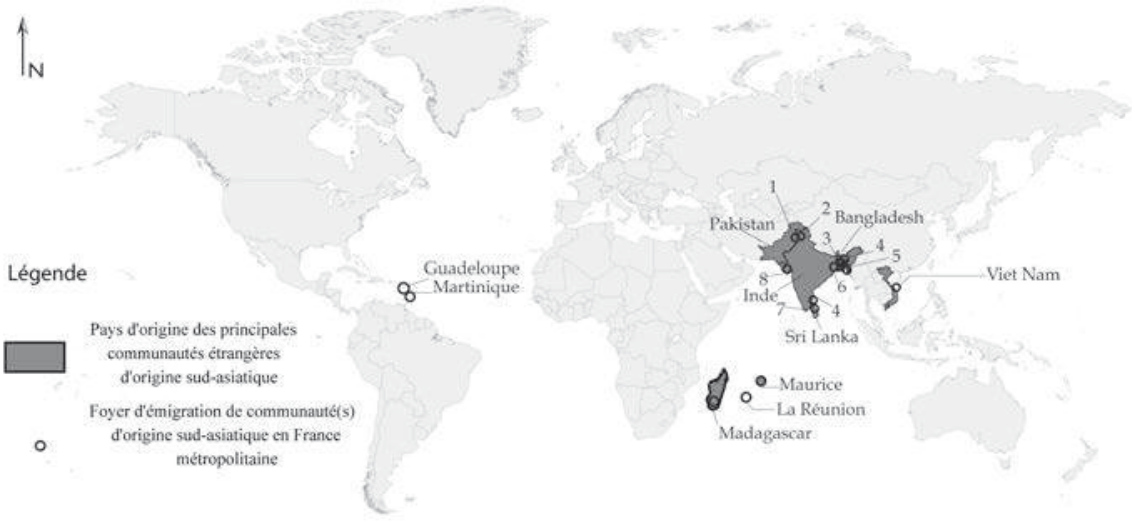

/// Figure 2 : La répartition des associations tamoules sri lankaises en région parisienne selon la localisation du siège social

\section{Légende}

Limite du départment

ws onn. Nom du départment

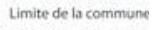

Cergy Nom de la commune

Commune avec au
moins une associatior tamoule

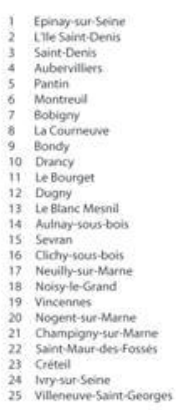

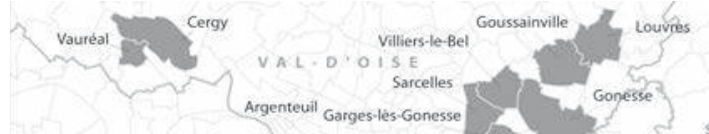

SEINE SAINT. DENIS Chelles

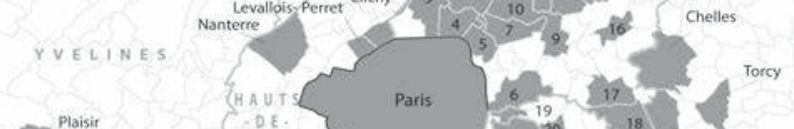

$S E I N E$ E.T. $M A R N=$

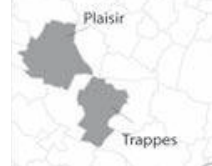




\section{Les transformations des trajectoires migratoires}

Lévolution des stratégies résidentielles en Île-deFrance des migrants d'origine sud-asiatique est liée aux transformations des trajectoires migratoires survenues depuis les années 1970. En effet, les types de logements occupés dépendent de l'étape du cycle migratoire et de la situation familiale des migrants ${ }^{11}$.

Dans le cas des immigrés venus d'Asie du Sud, il est possible de distinguer une première phase migratoire masculine. Les primo-arrivants sri lankais ou pakistanais venus dans les années 1970 et 1980 ont souvent loué (ou sous-loué) des petits appartements vétustes qu'ils partageaient avec plusieurs autres compatriotes. Le partage du logement à plusieurs permet à ces personnes d'économiser de l'argent malgré leur faible salaire. Cet argent est généralement en partie envoyé pour aider la famille restée au pays. Le reste constitue un capital qui permettra par la suite d'investir dans un logement ou de crééer une activité commerciale.

Les immigrés tamouls sri lankais arrivés depuis les années 1990 sont moins confrontés à cette réalité. Ils sont généralement hébergés par des membres de leur famille déjà installés depuis plusieurs années, voire des décennies, à Paris ou en banlieue. Aujourd'hui, ce sont surtout les immigrés bangladeshis, pour beaucoup clandestins, qui se retrouvent dans cette situation. Les membres de cette communauté, qui ont immigré plus tardivement en France, ne disposent généralement pas de relais familiaux pour les soutenir. Ils comptent souvent sur la solidarité communautaire pour trouver un lit dans un logement à partager avec d'autres Bangladeshis.

La venue des femmes est à l'origine du passage à la deuxième étape de la phase migratoire. La perspective de la vie familiale pousse les Sud-Asiatiques à rechercher un logement individuel, dans lequel les femmes ne seraient pas confrontées à la promiscuité avec d'autres hommes. Cette deuxième phase, qui suit la régularisation et le processus de regroupement familial, a commencé plus tôt, principalement depuis les années 1990 pour les courants migratoires plus anciens (sri lankais, pakistanais, indiens). Le temps d'attente pour obtenir un logement social décourage souvent les immigrés d'origine sud-asiatique. En outre, c'est seulement en 1998 que les familles de réfugiés et d'apatrides ont eu droit au regroupement familial sans condition de ressources et de logement. Ainsi, de nombreux réfugiés sri lankais ont préféré trouver un logement dans le secteur privé pour répondre L'évolution des stratégies résidentielles en île-de-France des migrants d'origine sud-asiatique est liée aux transformations des trajectoires migratoires survenues depuis les années 1970. aux impératifs administratifs afin de faire venir au plus tôt leurs proches. Dans la mesure du possible, les hommes cherchent un logement à Paris pour accueillir leur famille, avant de décider d'acquérir à crédit un appartement plus grand ou un pavillon en banlieue ${ }^{12}$. Enfin, la politique d'éradication des habitats insalubres de la Ville de Paris a eu pour conséquence, à partir de 2001, de baisser l'offre de logements bon marché dans le XVIII arrondissement. Le processus de gentrification a, à l'inverse, augmenté le prix du foncier et participé au déplacement en banlieue des immigrés d'origine sud-asiatique qui s'y trouvaient.

\section{Les deux ethnoterritoires sud-asiatiques de Paris}

L'évolution des stratégies résidentielles des groupes d'origine sud-asiatique a abouti depuis le milieu des années 1990 à une dissociation entre le lieu de résidence et les lieux d'approvisionnement. En effet, les deux anciens lieux d'implantation des communautés d'origine sud-asiatique le long de la rue du Faubourg-Saint-Denis sont deve- 
nus des ethnoterritoires commerciaux et surtout des centres minoritaires. Deux ethnoterritoires sud-asiatiques, La Chapelle et gare de l'Est, situés dans les $\mathrm{X}^{\mathrm{e}}$ et XVIII arrondissements, le long de la rue du Faubourg-Saint-Denis, sont considérés comme les quartiers indiens de Paris. Ces deux ethnoterritoires sont associés à deux ensembles culturels distincts : un quartier,

Deux ethnoterritoires sud-asiatiques, La Chapelle et gare de l'Est, situés dans les $X^{e}$ et XVIII arrondissements, le long de la rue du Faubourg. Saint-Denis, sont considérés comme les quartiers indiens de Paris.

Bangladesh) et de l'île Maurice. Ces deux quartiers sud-asiatiques sont exemplaires du phénomène de centralité minoritaire. À des degrés différents, ces deux ethnoterritoires sont, pour les populations d'origine sud-asiatique de la région parisienne, un espace de travail, d'échanges commerciaux et de sociabilisation. La présence déambulatoire de nombreuses personnes d'origine sud-asiatique dans ces deux quartiers et les produits ethniques proposés dans les commerces participent à renforcer, aux yeux des autres populations de la ville, l'“indianité" et la singularité de ces deux territoires par rapport aux autres espaces urbains de la capitale.

\section{La Chapelle, le Little Jaffna de Paris}

Le quartier de La Chapelle est situé à la limite entre les $\mathrm{X}^{\mathrm{e}}$ et XVIII arrondissements de Paris autour de la station de métro La Chapelle. La présence prépondérante de l'empreinte tamoule sri lankaise dans cet espace est à l'origine du surnom de Little Jaffna $^{13}$ donné à cet ethnoterritoire. Deux zones peuvent être distinguées à La Chapelle (figure 3).
La première, qui sétend au sud du boulevard de La Chapelle, est caractérisée par une forte concentration d'établissements commerciaux ou de services qui répondent en priorité aux besoins de la communauté tamoule. L'inscription spatiale des éléments culturels tamouls se matérialise par l'omniprésence de l'alphabet tamoul sur les vitrines des magasins ou sur les affiches collées sur les murs (avis nécrologiques, affiches de spectacles ou de manifestations culturelles ou politiques tamoules). En outre, de nombreux magasins portent un nom faisant référence à des villes tamoules du Sri Lanka (Jaffna/Yalpanam) ou d'Inde (Chennai/Madras, Pondichéry). Enfin, les chansons du cinéma Kollywood ${ }^{14}$ jouées dans les commerces du quartier sont des marqueurs sonores qui confirment que La Chapelle est un ethnoterritoire tamoul. Les seuls commerces sud-asiatiques qui ne soient pas détenus par des Tamouls dans cet espace sont les boucheries, les épiceries ou des salons de thé, qui appartiennent surtout à des musulmans du Sri Lanka ou à des Cingalais.

La Chapelle est ainsi un centre d'approvisionnement privilégié pour les Tamouls, mais également pour les autres communautés d'origine sudasiatique, grâce à la diversité des entreprises. En effet, le quartier compte des restaurants, de nombreuses épiceries proposant un choix très varié de produits du sous-continent indien, des bijouteries, des agences de voyages, des librairies tamoules, une école de conduite, une agence immobilière, des magasins de vidéos, des salons de coiffure (principalement pour hommes) et des salons de beauté (pour femmes) qui cherchent avant tout à répondre aux besoins d'une clientèle d'origine sud-asiatique.

La deuxième zone de La Chapelle, qui s'étend au nord du boulevard de la Chapelle, est essentiellement connue pour sa fonction religieuse avec la présence de trois temples hindouistes surtout fréquentés par des Tamouls. L’un est dédié à Ganesh, un autre à Maryammâ et le dernier à Sri Pathira- 


\section{Figure 3 : \\ Le quartier de La Chapelle ou le Little Jaffna français}

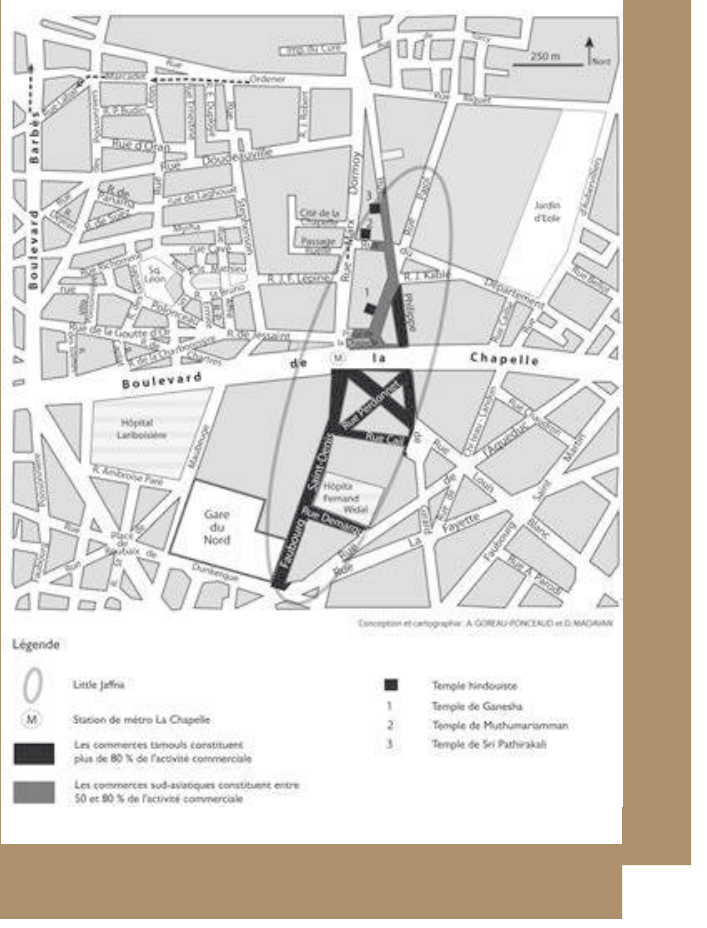

Conception et cartographie

A. Goreau-Ponceaud et D. Madavan.

kali. Le plus ancien, le temple de Ganesh, fondé en 1985, est le seul à avoir obtenu de la Mairie, depuis 1996, l'autorisation d'organiser une procession en l'honneur du dieu à la tête d'éléphant dans les rues de La Chapelle. Cette procession annuelle, organisée le premier dimanche de septembre, est devenue un événement populaire très attendu par des milliers de Parisiens, dont de nom- breux non-Tamouls. La Chapelle est aussi le centre du réseau social et de solidarité pour les Tamouls, en particulier pour les Sri-Lankais. C'est un lieu de rencontres avec d'autres membres de la communauté, où les migrants récemment arrivés, qui ne maîtrisent pas le français et qui nont pas de titre de séjour, peuvent obtenir des renseignements sur les démarches administratives et de l'aide pour trouver un emploi ou un logement. La Chapelle est ainsi, pour les Tamouls sri lankais, "le centre d'un vaste réseau de solidarité ethnique dont la signification dépasse l'espace géographique de la visibilité tamoule $e^{15 "}$. En outre, le quartier a un rôle important dans la transmission de la culture et de l'identité tamoules pour les deuxième et troisième générations. Le quartier est aussi le lieu où les jeunes d'origine tamoule nés en France présentent leur culture tamoule et/ou sri lankaise à leurs amis d'autres origines par le biais de la nourriture ou des vêtements.

Enfin, l'influence du Liberation Tigers of Tamil Eelam (LTTE) ${ }^{16}$ à La Chapelle est incontestable et confère au quartier une fonction politique. Ainsi, de nombreux magasins ont un portrait du défunt chef historique du LTTE, Prabhakaran, ou le drapeau du mouvement séparatiste. De nombreuses institutions culturelles et sociales liées au LTTE ont leur siège dans le quartier (Tamil Youth Organisation, Tamil Cholai, etc.). Les magasins de La Chapelle affichent aussi, de gré ou de force, des bannières noires pour commémorer la mort des dirigeants séparatistes LTTE (Prabhakaran, Balasingham, Thamilselvam), ou des bannières rouge et jaune (couleurs associées à l'État indépendant que les Tamouls souhaitent créer : le Tamil Eelam)

15. Gaëlle Dequirez, op. cit. 16. Liberation Tigers of Tamil Eelam (LTTE) est un mouvement indépendantiste fondé en 1976 par Velupillai Prabhakaran, dont le but affiché est de défendre les Tamouls du Sri Lanka. Pour cela, l'organisation demande le droit à l'autodétermination et la création d'un État, le Tamil Eelam, dans le nord et l'est de l'île. Pour obtenir leur indépendance, les Tigres se sont engagés dans une lutte armée avec le gouvernement central. Le LTTE s'est affirmé depuis 1987 comme l'acteur incontournable de la scène politique nationale. En effet, le mouvement a réussi à faire face aux différents assauts de l'armée gouvernementale et même aux forces indiennes qui ont tenté un temps de lui faire déposer les armes par la force. La défaite militaire des Tigres du 17 mai 2009 a ébranlé le mouvement. Son leader historique, Velupillai Prabhakaran, est mort et le LTTE a perdu toutes ses possessions territoriales dans l'île. Néanmoins, le mouvement séparatiste compte encore un important soutien dans la diaspora tamoule et n'a pas abandonné son rêve de créer un État indépendant pour les Tamouls du Sri Lanka. 
lors des célébrations du mouvement séparatiste (Maaveerar $\mathrm{Naal}^{17}$ ). C'est aussi dans ce quartier que de nombreuses manifestations ont été organisées pour soutenir les Tigres et dénoncer les opérations militaires de l'armée sri lankaise pendant l'offensive militaire finale en 2009, ou encore la détention des survivants tamouls dans des camps d'internement placés sous l'autorité de l'armée sri lankaise $\mathrm{e}^{18}$. Cette emprise du mouvement séparatiste à La Chapelle est d'ailleurs perçue comme dangereuse par les autorités françaises. Ces dernières ont conduit des raids de police dans le quartier afin d'arrêter des partisans du LTTE accusés de racketter des Tamouls sri lankais en France pour financer le mouvement séparatiste. La fonction politique de La Chapelle identifie clairement ce quartier comme un territoire diasporique tamoul pro-LTTE.

\section{Le quartier indien de gare de l'Est}

Le deuxième quartier sud-asiatique de Paris se situe également le long de la rue du Faubourg-Saint-Denis, dans le $\mathrm{X}^{\mathrm{e}}$ arrondissement, entre la rue Lafayette et la rue de l'Échiquier (figure 4). Plusieurs communautés d'origine sud-asiatique cohabitent dans cet ethnoterritoire où dominent les cultures des régions septentrionales du sous-continent indien (nord de l'Inde, Pakistan, Bangladesh) et de l'̂̂le Maurice. Il est possible de distinguer deux espaces. Le premier correspond à la section de la rue du Faubourg-Saint-Denis comprise entre la rue La Fayette (qui est en quelque sorte la frontière entre les deux ethnoterritoires sud-asiatiques) et le boulevard Magenta. On y retrouve essentiellement de petites enseignes de restauration bon marché bangladeshies, pakistanaises et nord-indiennes.

\section{Figure 4 :}

\section{Le quartier sud-asiatique de gare de l'Est}

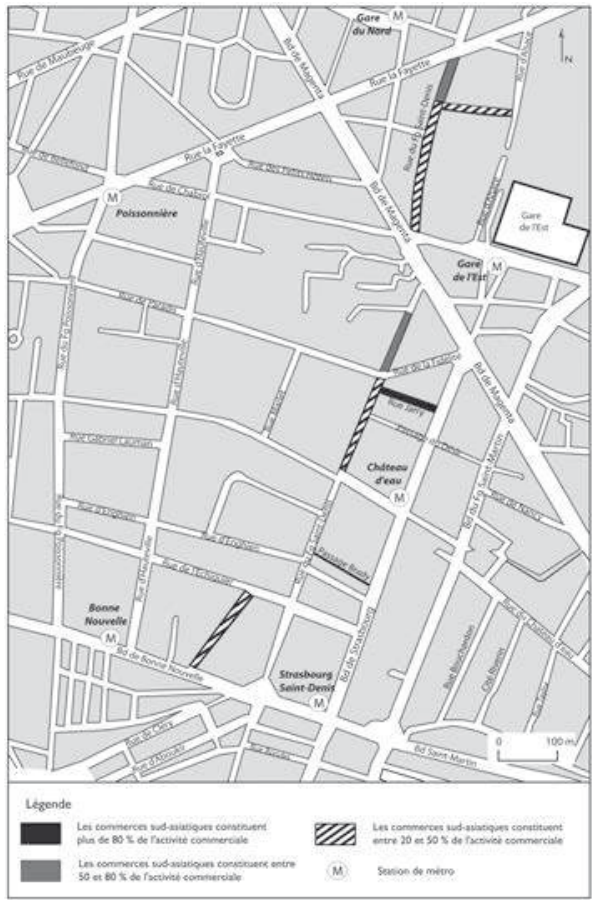

Source : Enquête de terrain, décembre 2013 Conception et réalisation, D. Madavan, 2014.

Ces commerces sont surtout fréquentés par des hommes originaires du même pays que le propriétaire et on y trouve très peu de femmes ou de clients qui ne soient pas d'origine sud-asiatique.

Le deuxième espace comprend la section de la rue du Faubourg-Saint-Denis située entre le boulevard Magenta et la rue de l'Échiquier et les petites rues perpendiculaires adjacentes. Les commerces sud- 
asiatiques de cet espace ont des enseignes faisant référence au Pakistan, à l'Inde et à l'île Maurice. On trouve surtout dans cette partie des restaurants, des épiceries, des agences de voyages et des magasins de DVD de musiques et de films de Bollywood qui répondent surtout aux goûts et aux besoins de ces communautés. Contrairement à l'espace précédent, la présence déambulatoire des personnes d'origine nord-indienne est moins importante même si elle est plus variée. Ce secteur attire plus de femmes d'origine sud-asiatique et des jeunes de la deuxième génération. Plusieurs commerces de ce secteur ont aussi une importante clientèle qui n'est pas d'origine sud-asiatique. Les restaurants du passage Brady ou le magasin de DVD Bollywood attirent de nombreux amoureux de l'Inde et de sa culture. Le quartier sud-asiatique de gare de l'Est a une fonction d'approvisionnement et de solidarité communautaire (renseignements, aide pour trouver du travail ou un logement) pour les Sud-Asiatiques non tamouls. Par contre, ce quartier ne semble pas avoir de fonction religieuse ou politique.

Contrairement à La Chapelle, ce secteur du quartier indien de gare de l'Est est un espace mixte où les personnes d'origine sud-asiatique cohabitent avec d'autres communautés. Ainsi, plus on s'approche de la rue de l'Échiquier, plus les commerces sud-asiatiques sont supplantés par des commerces turcs $^{19}$. Au niveau des petites rues adjacentes à cette portion de la rue du Faubourg-Saint-Denis, seuls le passage Brady et la rue de Jarry comptent une présence significative de commerces sud-asiatiques. Les autres rues comptent quant à elles surtout des établissements proposant des produits et des services destinés aux populations originaires d'Afrique subsaharienne. Lévolution de la situation de la rue de la Fidélité est très significative. Le dynamisme des entepreneurs originaires d'Afrique noire s'est traduit par l'ouverture au cours de ces dernières années de salons de coiffure, de cafés et de restaurants dans la rue, alors qu'à l'inverse les commerces sud-asiatiques y ferment. Le caractère indien du quartier tend à s'effacer.

La baisse d'attractivité du quartier indien de gare de l'Est pousse certains Nord-Indiens, Pakistanais, Mauriciens ou Bangladeshis à préférer se rendre à La Chapelle pour faire leurs courses ou boire un thé. Néanmoins, de nombreuses personnes d'origine sud-asiatique (non tamoules) interviewées affirment qu'elles ne considèrent pas pour autant La Chapelle comme leur quartier.

\section{Conclusion}

L'installation des premières vagues de populations d'origine sud-asiatique à Paris s'est accompagnée de la constitution, dans les Xe et XVIII arrondissements, de deux ethnoterritoires sud-asiatiques distincts : un quartier dominé par les cultures des régions septentrionales du sous-continent indien et de l'île Maurice (gare de l'Est) et un quartier dominé par la culture tamoule (La Chapelle). Les régularisations et le processus de regroupement familial ont durablement transformé leurs stratégies résidentielles. Les membres de ces communautés ont, pour beaucoup, préféré quitter la capitale pour s'installer dans les villes de la banlieue nordest de Paris. Malgré cette dissociation entre le lieu de résidence et les lieux d'approvisionnement, La Chapelle et gare de l'Est demeurent des ethnoterritoires commerciaux et surtout des centres minoritaires (commercial, culturel, social et de solidarité, etc.) pour les différentes communautés d'origine sud-asiatique vivant en Île-de-France. 